\title{
Co-prescription of $\mathrm{H} 2$ receptor blockers and proton pump inhibitors with warfarin in general practice
}

\author{
A Pali S Hungin, Greg P Rubin, Hilda O'Flanagan
}

\begin{abstract}
Summary
Acid-suppressing drugs and anticoagulants are used increasingly in general practice. Warfarin is potentiated by some acid-suppressing drugs, notably cimetidine and omeprazole, through interference of the cytochrome P450 system. This study aimed to ascertain the extent of co-prescribing of warfarin and acidsuppressing drugs in general practice. We conducted a retrospective survey of the records of all patients prescribed acidsuppressing drugs over a 2-year period to ascertain those who had also taken warfarin; we also made a cross-sectional survey of all patients on warfarin to ascertain those who had taken acid-suppressing drugs. From a general practice population of 45574 patients in northern England, $3423(7.5 \%)$ had been prescribed acidsuppressing drugs during the previous 24 months. Of 274 patients who had been on warfarin, $44(16.1 \%)$ had also taken acidsuppressing drugs (26 $\mathrm{H} 2$ receptor blockers and 18 proton-pump inhibitors). The commonest reasons for anticoagulation were thrombo-embolic disease $(40.9 \%)$, atrial fibrillation (36.4\%), valvular heart disease $(\mathbf{1 8 . 2 \%})$, and surgical prophylaxis $(4.5 \%)$. The indications for concurrent acid-suppressing drugs were: 'dyspepsia' $38.6 \%$, reflux $22.7 \%$, oesophagitis $13.6 \%$, duodenal ulcer $13.6 \%$, gastric ulcer $4.5 \%$, unknown $6.8 \%$. There have been no studies from primary care to evaluate the possible clinical effects of the concomitant use of acid-suppressing drugs and warfarin; some fluctuations in coagulation control, particularly in patients taking the combinations intermittently, may be due to this.
\end{abstract}

Keywords: H2 receptor blockers; acid-suppressing drugs; warfarin; proton pump inhibitors

Department of Primary Health Care, The Medical School, University of

Newcastle upon Tyne, NE2, UK

G P Rubin

The Health Centre, Eaglescliffe, Stockton on Tees TS16 9EA, UK H O'Flanagan

Accepted 5 July 1999 tidine, ranitidine, nizatidine) and proton pump inhibitors (PPIs, eg, omeprazole, lanzoprazole), and anticoagulants, benzodiazepines, phenytoin, theophyllines and calcium channel blockers. ${ }^{1}$ The potential for interaction between PPIs and warfarin is of particular concern because of their increasing use and practical considerations for anticoagulation control. Studies have indicated that $1.26 \%$ of the UK general practice patients are on long-term acidsuppression therapy ${ }^{2}$ and $0.45 \%$ are on longterm PPIs. ${ }^{3}$ The number of patients on warfarin has increased following recommendations about stroke prevention in patients with atrial fibrillation. Although the clinical significance of the interaction between warfarin and some ASDs is uncertain, the potential for problems needs highlighting. The purpose of this study was to ascertain the extent of and the reasons for this co-prescription in UK general practice.

\section{Methods}

The study consisted of a retrospective survey of the records of patients who had been prescribed acid-suppressing drugs (H2 receptor blockers and PPIs) in the 2 years to January 1998 to ascertain those who had also been prescribed warfarin, and a cross-sectional survey of all patients on warfarin to ascertain those who had been prescribed ASDs concurrently. This dual approach was used to maximise the ascertainment of patients who had been prescribed this dual therapy.

The study was set in three general practices in northern England, representing a mix of urban and suburban locations. The combined patient population was 45574 , served by 21 general practitioners. All the practices maintained their prescribing records on similar computer systems and also held registers of patients on warfarin.

Case finding was done by an initial search of the computerised prescribing records using pre-determined codes, followed by a trawl of the paper records. All prescriptions for ASDs where warfarin had also been prescribed were identified. This enabled continuous as well as one-off ASD prescribing to those on warfarin to be detected. Checks were made for concurrence of prescribing and data entered into a database created using EpInfo software. A system of double data entry was used to eliminate transcription errors and $10 \%$ of all final entries were audited against the clinical records. This confirmed that no systematic errors had occurred and that the data were accurate. 
Results

PATIENTS AND PRESCRIBING

From a registered population of 45574 patients, $3423(7.5 \%)$ had been prescribed ASDs during the previous 2 years, comprising 1256 patients $(36.6 \%)$ on cimetidine, 772 $(22.5 \%)$ on ranitidine, $17(0.5 \%)$ on famotidine, six $(0.2 \%)$ on nizatidine, $887(25.9 \%)$ on omeprazole, and $485(14.1 \%)$ on lansoprazole.

A total of 274 ( $0.6 \%$ of the study population) had been prescribed warfarin. Of these, 44 $(16.1 \%)$ had taken ASDs concurrently, comprising $26(9.5 \%)$ patients on $\mathrm{H} 2$ receptor blockers and $18(6.6 \%)$ on PPIs. The combinations with warfarin were: 20 patients on ranitidine $(45.4 \%), 11$ on omeprazole $(25 \%)$, seven on lansoprazole $(15.9 \%)$ and six on cimetidine $(13.6 \%)$.

The sex ratio of patients on combined prescriptions was $1: 1$; the mean age was 65 years (males 62 years, range 35-79; females 69 years, range $42-83$ ); and $61 \%$ of patients were aged 65 or over.

REASONS FOR PRESCRIBING

Reasons for warfarin use in the 44 patients taking both warfarin and an ASD were 18 with thrombo-embolic disease $(40.9 \%), 16$ with atrial fibrillation $(36.4 \%)$, eight with valvular heart disease $(18.2 \%)$, and two with surgical prophylaxis $(4.5 \%)$.

One of the more common reasons for the concurrent prescription of ASDs (table) was gastro-oesophageal reflux disease (GORD). Of those patients on PPIs, $55.6 \%$ were being treated for GORD compared with $23 \%$ of those on $\mathrm{H} 2$ receptor blockers.

PATTERNS OF PRESCRIBING

Continuous acid-suppression therapy had been taken by $65.9 \%$ of warfarinised patients. Of the remainder, $20.5 \%$ had received a single prescription only and $13.6 \%$ short (usually monthly) intermittent courses of ASDs. Continuous therapy was more likely with $\mathrm{H} 2$ receptor blockers $(73.1 \%$ of all patients on combined prescriptions) than with PPIs $(55.6 \%)$.

Table Reasons for concurrent ASDs in patients on warfarin

\begin{tabular}{lc}
\hline & $n(\%)$ \\
\hline 'Dyspepsia', unspecified & $17(38.6)$ \\
Gastro-oesophageal reflux & $10(22.7)$ \\
Oesophagitis & $6(13.6)$ \\
Duodenal ulcer & $6(13.6)$ \\
Gastric ulcer & $2(4.5)$ \\
Other & $3(6.8)$ \\
\hline
\end{tabular}

1 Stockley I H. Drug interactions. London: Blackwell Scientific Publications, 1994.

2 Rubin GP, Contractor B, Bramble MG. The use of long term acid suppression therapy. Br f Clin Pract 1995;49:11920 .

3 Hungin APS, Rubin GP, O'Flanagan H. Long term prescribing of proton pump inhibitors in general practice: clinical and cost implications (Abstract). Gastroenterology 1997;112:A19.

\section{Discussion}

The study determined the extent and reasons for the co-prescribing of warfarin and ASDs in general practice. It did not evaluate any clinical effect from potential interactions. There were several reasons for this, but it was chiefly due to the fact that variations in anticoagulation control are difficult to link to cause. Even in the absence of a recognised interaction, anticoagulation control is influenced by a number of other confounding factors, eg, compliance or alcohol intake. Also, the control range for individual patients varies with the underlying disorder for which warfarin is prescribed. Thus, variations in anticoagulation test results or warfarin dose could not be considered as outcome measures to assess the effect of an interaction in a general practice setting.

Whilst the practical significance of the interaction between some ASDs and warfarin remains unclear, their co-prescription appears to be an established part of clinical practice. In this study ASDs were prescribed to $16.1 \%$ of warfarinised patients over a 2-year period. Omeprazole, lansoprazole and cimetidine comprised a large proportion of these, despite the fact that the Association of the British Pharmaceutical Industry data sheets allude to the risk of interactions. Pantoprazole, which appears to avoid these interactions, did not feature in this study as it had not been marketed at that stage. As increasing numbers of patients are diagnosed as having both GORD and atrial fibrillation, the requirement for such co-prescribing is likely to increase, particularly in the elderly, who are more susceptible to the adverse effects of polypharmacy.

There are no data highlighting any practical management difficulties in patients in general practice. Studies with omeprazole and lansoprazole in healthy volunteers have indicated changes in warfarin bioavailability, ${ }^{45}$ but there is no clear evidence on the effect on clotting. Whilst the overall safety of the PPIs has been established it seems that some products have fewer interactions than others. ${ }^{6}$ The continuous co-prescription of these drugs with warfarin may well result in a steady-state anticoagulation interference but their intermittent use could explain some of the fluctuations in control seen during anticoagulation monitoring. Clinicians should be aware of possible interactions and should consider the use of ASDs known not to interfere with the cytochrome P450 system where co-prescription is deemed necessary.

We would like to thank the GPs and staff at the Tennent Street, Thornaby and Eaglescliffe Health Centres and particularly Dr John Nicholas, for access to records. The study was done through the Northern Primary Care Research Network.

\footnotetext{
4 Unge P, Svedberg LE, Nordgren A, et al. A study of the interaction of omeprazole and warfarin in anticoagulated patients. Br f Clin Pharmacol 1992;34:509-12.

5 Sutfin T, Balmer K, Bostrom H, Eriksson S, Hoglund P, Paulsen O. Stereoselective interaction of omeprazole with Paulsen O. Stereoselective interaction of omeprazole with warfarin in

6 Arnold R. Safety of proton pump inhibitors - an overview. Aliment Pharmacol Ther $1994 ; 8(\mathrm{~S} 1): 65-70$.
} 